\title{
Análisis crítico de un artículo: La terapia intensiva con atorvastatina tiene un beneficio marginal en pacientes con enfermedad coronaria estable
}

\author{
Claudio Puebla $A^{1,2}$, Cristian Vergara $M^{1 a}$, \\ Roberto Vallejos $\mathrm{Pla}^{1 a}$, Andrés Pizarro $\mathrm{C}^{\mathrm{la}}$, \\ Francisco Dodds $B^{1 a}$, Cristian G odoy $T^{1 a}$.
}

\section{Critical appraisal: Intensive lipid lowering with atorvastatin in patients with stable coronary disease.}

LaRosa JC, Grundy SM, Waters DD, Shear $C$, Barter P, Fruchart JC, Gotto AM, G reten $H$, Kastelein JJ, Shepherd J, Wenger NK; Treating to New Targets (TNT) Investigators. TNT study. N Engl J Med 2005; 352: 1425-35.

\begin{abstract}
Previous trials have demonstrated that lowering lowdensity lipoprotein (LDL) cholesterol levels below currently recommended levels is beneficial in patients with acute coronary syndromes. We prospectively assessed the efficacy and safety of lowering LDL cholesterol levels below $100 \mathrm{mg}$ per deciliter (2.6 mmol per liter) in patients with stable coronary heart disease (CHD). Methods: A total of 10,001 patients with clinically evident CHD and LDL cholesterol levels of less than $130 \mathrm{mg}$ per deciliter (3.4 mmol per liter) were randomly assigned to double-blind therapy and received either $10 \mathrm{mg}$ or $80 \mathrm{mg}$ of atorvastatin per day. Patients were followed for a median of 4.9 years. The primary end point
\end{abstract}

${ }^{1}$ Servicio de Medicina Interna, Hospital Naval "Almirante Nef", Viña del Mar.

${ }^{2}$ Departamento de Salud Pública, Universidad de Valparaíso. Valparaíso, Chile.

${ }^{a}$ Residente de Medicina Interna, Universidad de Valparaíso.

Correspondencia a: Dr. Claudio Puebla A. E mail: cpuebla@entelchile.net 
was the occurrence of a first major cardiovascular event, defined as death from CHD, nonfatal non-procedure-related myocardial infarction, resuscitation after cardiac arrest, or fatal or nonfatal stroke. Results: The mean LDL cholesterol levels were $77 \mathrm{mg}$ per deciliter $(2.0 \mathrm{mmol}$ per liter) during treatment with $80 \mathrm{mg}$ of atorvastatin and $101 \mathrm{mg}$ per deciliter (2.6 mmol per liter) during treatment with $10 \mathrm{mg}$ of atorvastatin. The incidence of persistent elevations in liver aminotransferase levels was 0.2 percent in the group given $10 \mathrm{mg}$ of atorvastatin and 1.2 percent in the group given $80 \mathrm{mg}$ of atorvastatin $(\mathrm{P}<0.001)$. A primary event occurred in 434 patients (8.7 percent) receiving $80 \mathrm{mg}$ of atorvastatin, as compared with 548 patients (10.9 percent) receiving $10 \mathrm{mg}$ of atorvastatin, representing an absolute reduction in the rate of major cardiovascular events of 2.2 percent and a 22 percent relative reduction in risk (hazard ratio, 0.78; 95 percent confidence interval, 0.69 to 0.89 ; $\mathrm{P}<0.001$ ). There was no difference between the two treatment groups in overall mortality. Conclusions: Intensive lipid-lowering therapy with $80 \mathrm{mg}$ of atorvastatin per day in patients with stable CHD provides significant clinical benefit beyond that afforded by treatment with $10 \mathrm{mg}$ of atorvastatin per day. This occurred with a greater incidence of elevated aminotransferase levels.

\section{CONCLUSIÓN DE LOS REVISORES:}

El uso de terapia intensiva con atorvastatina ( $80 \mathrm{mg}$ día) reduce los infartos al miocardio (IM) no fatales y los accidentes vasculares encefálicos (AVE) fatales y no fatales, pero no afecta la mortalidad global en comparación con dosis de $10 \mathrm{mg}$ de atorvastatina en pacientes con enfermedad coronaria estable.

\section{PREgunta CLÍNICA:}

En pacientes con enfermedad coronaria estable, ¿ el uso de altas dosis de atorvastatina (80 $\mathrm{mg} /$ día) reduce eventos cardiovasculares en comparación a dosis bajas de atorvastatina (10 $\mathrm{mg} /$ día)?

\section{Contexto:}

El uso de estatinas ha demostrado beneficios, tanto para la prevención primaria en pacientes de alto riesgo ${ }^{1}$, como para la prevención secundaria de enfermedad cardiovascu$\operatorname{lar}^{2}$. Las recomendaciones actuales han fijado como meta un $\mathrm{LDL}<100 \mathrm{mg} / \mathrm{dl}$ en pacientes con enfermedad coronaria ${ }^{3}$, sin embargo, existe evidencia inicial, para sugerir que valores menores de LDL pueden aumentar los beneficios sin aumentar significativamente los efectos secundarios de las estatinas.

\section{CARACTERÍSTICAS DEL ESTUDiO:}

Tipo de estudio: Randomizado, multicéntrico. Seguimiento: 4,9 años. Pacientes: Entre 35 y 75 años, con enfermedad coronaria definida por uno de los siguientes criterios: IM previo, angina previa o actual con evidencias de enfermedad coronaria objetiva, previa revascularización. Se excluyeron pacientes ${ }^{4}$ con hipersensibilidad a las estatinas, enfermedad hepática (transaminasas $>1,5$ veces el valor máximo normal), mujeres embarazadas o en lactancia, síndrome nefrótico, diabetes, hipotiroidismo o hipertensión no controladas, cuadro coronario agudo o revascularizados a menos de 1 mes del screening del paciente, fracción de eyección $<30 \%$, enfermedad valvular significativa, limitación a la absorción de la droga, creatinfosfokinasa (CPK) total $>6$ veces el valor máximo normal, abuso de alcohol, 
otras enfermedades crónicas con expectativa de vida limitada, uso concomitante de inmunosupresores. Los pacientes elegibles fueron 18.469, se excluyeron 3.005 y luego de un periodo de run-in (8 semanas con $10 \mathrm{mg}$ de atorvastatina) se excluyeron otros 5.461 . Finalmente se randomizaron 10.003 , pero se analizaron 10.001 pacientes. Intervención: 4.960 pacientes recibieron $80 \mathrm{mgr}$ de atorvastatina (dosis alta). Comparación: 4.968 pacientes recibieron $10 \mathrm{mgr}$ de atorvastatina (dosis baja). Outcomes: Primario: eventos cardiovasculares mayores (definidos como: muerte por enfermedad coronaria o IM no fatal o paro cardiorrespiratorio recuperado o AVE fatal-no fatal). Secundarios: AVE, hospitalización por insuficiencia cardíaca congestiva, enfermedad vascular periférica, muerte de cualquier causa, cualquier evento cardiovascular, cualquier evento coronario. Aparición de efectos secundarios.

\section{VALIDEZ:}

Randomizado:

Ocultamiento de secuencia de aleatorización:

Seguimiento:

Análisis con intención de tratar:

Fueron ciegos a la intervención:

Pacientes

Tratantes

Recolectores de datos

Adjudicadores de eventos

Analistas de datos

Grupos similares respecto a variables pronósticas conocidas:

\section{SI}

No consignado 99,3\%

SI

SI

SI

No consignado

SI

SI

SI

Resultados:

\begin{tabular}{|c|c|c|c|c|}
\hline Evento & $\begin{array}{l}\text { Tasa eventos grupo } \\
\text { atorvastatina } 80 \mathrm{mg}\end{array}$ & $\begin{array}{l}\text { Tasa eventos grupo } \\
\text { atorvastatina } 10 \mathrm{mg}\end{array}$ & $\begin{array}{c}\text { RRR } \\
95 \% \text { IC }\end{array}$ & $\begin{array}{c}\text { NNT } \\
95 \% \text { IC }\end{array}$ \\
\hline Outcome primario & $8,8 \%$ & $11 \%$ & $\begin{array}{c}20 \% \\
(10 \text { a } 29 \%)\end{array}$ & $\begin{array}{c}46 \\
(30 \text { a } 97)\end{array}$ \\
\hline $\mathrm{IM}$ & $4,9 \%$ & $6,2 \%$ & $\begin{array}{c}21 \% \\
(7 \text { a } 33 \%)\end{array}$ & $\begin{array}{c}77 \\
(45 \text { a } 250)\end{array}$ \\
\hline AVE no fatal & $2,4 \%$ & $3,1 \%$ & $\begin{array}{c}25 \% \\
(5 \text { a } 41 \%)\end{array}$ & $\begin{array}{c}130 \\
(71 \text { a } 778)\end{array}$ \\
\hline $\begin{array}{l}\text { Mortalidad } \\
\text { cardiovascular }\end{array}$ & $2,5 \%$ & $3.1 \%$ & $\begin{array}{c}20 \% \\
(-3 \text { a } 39 \%)\end{array}$ & $\begin{array}{l}192 \\
\text { (NS) }\end{array}$ \\
\hline $\begin{array}{l}\text { Mortalidad } \\
\text { global }\end{array}$ & $5,7 \%$ & $5,7 \%$ & $0 \%$ & NS \\
\hline RAM* & $8,2 \%$ & $5,8 \%$ & $\begin{array}{c}\text { ARR } \\
41 \% \\
(22 \text { a } 64 \%)\end{array}$ & $\begin{array}{c}\mathrm{NNH} \\
42 \\
(29 \text { a } 72)\end{array}$ \\
\hline
\end{tabular}

RRR $=$ reducción de riesgo relativo. $\mathrm{NNT}=$ número necesario de tratar. $\mathrm{IC}=$ intervalo de confianza. $\mathrm{ARR}=$ aumento relativo del riesgo. $\mathrm{NNH}=$ número necesario para hacer daño. NS = No significativo.

${ }^{*} \mathrm{RAM}=$ Reacción adversas a medicamentos. 


\section{COMENTARIOS Y APLICACIÓN PRÁCTICA:}

- La evaluación de la validez no muestra limitaciones severas, más aún incluye un número grande de pacientes, con un seguimiento notable para el tipo de estudio. Los mayores problemas están en su diseño, lo que puede limitar su aplicabilidad. Todos los pacientes tuvieron un período de run in, en el que se daba un tratamiento de $10 \mathrm{mg}$ de atorvastatina por día durante 8 semanas, que excluyó a más de 5 mil pacientes, por mala tolerancia, adherencia o la aparición de efectos indeseables. Los pacientes finalmente randomizados, tenían menor riesgo de desarrollar complicaciones, lo que puede subestimar la tasa de efectos secundarios. Tampoco se definió claramente varios de éstos antes de iniciar el estudio.

- Respecto a la mortalidad, hubo una tendencia a la disminución en las relacionadas con enfermedad coronaria, pero no hubo efecto en la mortalidad de cualquier causa. Esta pequeña reducción fue compensada con un aumento no significativo de muerte asociada a cáncer y otras enfermedades no traumáticas. Hay que evaluar con cuidado dichos resultados, porque pueden representar un riesgo a futuro en poblaciones mayores y con algún factor de riesgo.

- En este grupo de pacientes de riesgo moderado, se logra una reducción de eventos cardiovasculares (especialmente IM no fatal y AVE), sin afectar la mortalidad global, con un aumento leve de efectos secundarios (habiéndose filtrado ya la población de mayor riesgo).

- El costo de la intervención hace no recomendable la utilización de atorvastatina $80 \mathrm{mg} /$ día en todos los pacientes, reservando la indicación para pacientes de mayor riesgo cardiovascular.

- Otro aspecto a considerar es la gran participación que hubo de las empresas farmacéuticas en el desarrollo del estudio. Esta incluyó diseño, elaboración y análisis de los datos, dejándose a los autores la interpretación de los resultados y elaboración del manuscrito final. Todos los autores, además, han estado relacionados con la industria farmacéutica.

\section{REFERENCIAS}

1. Heart Protection Study Collaborative Group. MrC/BHF Heart Protection Study of cholesterol lowering with simvastatin in 20,536 high-risk individuals: a randomised placebo-controlled trial. Lancet 2002; 360: 7-22.

2. Scandanavian Simvastatin Survival Study Group. Randomised trial of cholesterol lowering in 4444 patients with coronary heart disease: the Scandinavian Simvastatin Survival Study (4S). Lancet 1994; 344: 1383-9.

3. Expert Panel on Detection, Evaluation, and Treatment of High Blood Cholesterol in Adults. Executive summary of Third Report of the National Cholesterol Education Program (NCEP) Expert Panel on Detection, Evaluation, and Treatment of High Blood Cholesterol in Adults (Adult Treatment Panel III). JAMA 2001; 285: 2486-97.

4. Waters D, Guyton J, Herrington D, McGowan M, Wenger N, Shear C. Treating to New Targets (TNT) Study: Does Lowering Low-Densitiy Lipoprotein Cholesterol Levels Below Currently Recommended Guidelines Yield Incremental Clinical Benefit? Am J Cardiol 2004; 93: 154-8. 